\title{
Resonant Interference Effect in Scattering of an Electron by an Electron in the Field of Two Pulsed Laser Waves
}

\author{
Elena A. Padusenko*, Alexandr A. Lebed, Sergei P. Roshchupkin \\ Institute of Applied Physics, National Academy of Sciences of Ukraine,58, Petropavlovskaya Str., Sumy, 40000 Ukraine \\ *Corresponding Author: lpadusenko@yandex.ru
}

Copyright (c) 2013 Horizon Research Publishing All rights reserved.

\begin{abstract}
Resonant scattering of an electron by an electron in the field of two unidirectional pulsed laser waves is studied theoretically. The interference kinematical region is considered. Stimulated emission and absorption of external waves' photons by electrons is correlated in the interference region. Resonance condition in the interference region is specified. Analytical expressions for the amplitude and differential cross section of the process for waves' circular polarization are obtained in the first order of the perturbation theory. The cross section contains resonant peaks, their altitude and width are determined by external waves parameters. Considerable effect of wave polarization on the character of stimulated emission and absorption of external waves' photons is found. It is demonstrated that the differential interference cross section of scattering of nonrelativistic electrons by each other in the field of two pulsed light waves of the optical frequency is approximately ten times greater in the magnitude than the corresponding one in the external field absence. Obtained results can be verified experimentally, for example, at the SLAC National Accelerator Laboratory (Stanford, USA) or in the frame of the FAIR Project (Darmstadt, Germany).
\end{abstract}

Keywords Field of Two Laser Pulsed Waves, Electron, Resonant Scattering, Interference Region, Correlated Emission and Absorption

\section{Introduction}

Widespread application of laser pulses [1] in both applied and fundamental research stimulates study of quantum electrodynamics (QED) processes in pulsed laser fields of different configurations. Nonlinear effects in force fields have been the object of scientific research for a long time already [2-9]. Experimental verification of QED effects in the laser field was carried out at the facility SLAC National Accelerator Laboratory (Stanford, USA) [10,11], and also is included into scientific program of FAIR international project based on the laser system PHELIX [12-13]. Development of new laser systems naturally results in improving of a model of description of modern lasers electromagnetic radiation [14-17]. The theoretical basis for QED processes study is the exact solution of the Dirac equation for an electron in the plane electromagnetic field, which is correct for a plane wave of arbitrary spectral composition [18,19].

Scattering of an electron by an electron is one of the fundamental QED processes. The cross section of this process in the external field absence was theoretically obtained by Möller [19,20]. In the presence of an external laser field this process is accompanied by stimulated emission and absorption of external field photons. Scattering of an electron by an electron in the field of a laser wave was studied in [5-8,17].

The theory of QED processes in the field of two laser waves was developed in [21-36], in the field of arbitrary number of plane monochromatic waves - in [37,38]. The interference quantum effect for scattering of an electron by an electron in the field of two or more plane monochromatic waves was discovered [24]. The essence of this effect is that emission and absorption of photons of external waves are correlated at a certain kinematics of the process. Study of effects caused by second pulsed laser wave presence is of interest due to the fact that probability of stimulated processes in the interference kinematics is generally greater than in other one.

Scattering of an electron by an electron in the field of a laser wave as a process of the second order in the fine structure constant can be resonant (Oleinik, [39,40]). Oleinik resonances are one of fundamental effects of QED in a laser field. The processes of the first order, which are forbidden by conservation laws in the external field absence, can occur in an electromagnetic wave. The resonant character relates to the fact that within a certain range of energy and momentum values a particle in an intermediate state may fall within the mass shell. Then a higher-order process is effectively reduced into two consecutive lower-order processes. Resonant cross sections of particles scattering can exceed the corresponding ones in the external field absence by several orders of the magnitude [5-7,17,39-49]. Thus, resonant scattering of an electron by an electron in the external light field was studied in [5-7,17,41-45]. 
It important to emphasize that when resonant conditions are satisfied the amplitude of particles scattering in the field of a plane monochromatic wave formally becomes infinite. Elimination of resonant infinity is usually performed by including of radiative corrections into the Green function of the intermediate particle by the Breit-Wigner procedure [39-44]. Elimination of the resonant infinity strictly mathematically becomes possible in the frame of the quasimonochromatic wave model, which can describe the field of the powerful pulsed laser up to the femtosecond range [6-7,17,45-49]. General processes (both resonant and nonresonant) of the both first and second order in the field of a single pulsed wave were studied in detail in [45-55], and results have been summarized in monographs $[6,17]$ and reviews $[7,8]$. Resonant scattering of a lepton by a lepton was studied in [45]. It is found that within a broad range of particle relative velocity resonant differential cross section of scattering of an electron by an electron (positron, muon) in the field of a single pulsed wave exceeds the corresponding cross section in the external field absence by several orders of the magnitude within the whole range of entrance polar angles.

The present paper is devoted to studying of the influence of two pulsed waves on resonant scattering of an electron by an electron in the interference region. The model of two pulsed plane waves allows us to describe the field of the two-mode pulsed laser, when laser radiation is characterized by presence of two frequency allotments in the field spectral distribution. At that the process is considered in the first order of the perturbation theory with respect to the interaction between electrons (the Born approximation), i.e. for sufficiently fast particles $\left(v_{j} / c \gg \alpha, v_{j}\right.$ is the electron velocity, $c$ is the light velocity in a vacuum, $\alpha$ is the fine structure constant). Thereby during the interaction time electrons do not have time to derivate significantly from the initial direction of movement at the expense of ponderomotive effect, consequently, spatial inhomogeneity in the transverse direction can be neglected [14]. The external light field is taken into account exactly.

The amplitude of the field strength of powerful ultra short laser pulses changes greatly in both space and time. Thus, we consider the field of two pulsed laser waves as two plane nonmonochromatic waves, propagating in the same direction. At that their planes of polarization belong to the plane $x y$, and the waves propagate along the $z$-axis. The four-potential of such a field has the form

$$
\begin{gathered}
A\left(\varphi_{1}, \varphi_{2}\right)=A_{01} \cdot g_{1}\left(\frac{\varphi_{1}}{\omega_{1} \tau_{1}}\right)+A_{02} \cdot g_{2}\left(\frac{\varphi_{2}}{\omega_{2} \tau_{2}}\right), \\
\varphi_{n}=k_{n} x=\omega_{n}(t-z / c), \quad n=1,2, \\
A_{0 n}\left(\varphi_{n}\right)=\frac{c F_{0 n}}{\omega_{n}}\left(e_{n x} \cos \varphi_{n}+\delta_{n} e_{n y} \sin \varphi_{n}\right) .
\end{gathered}
$$

The index $n$ labels the number of the laser wave. Each term in (1) corresponds to the field of the first and second wave, respectively. Here, $\varphi_{n}$ is the wave phase; $F_{0 n}$ is the strength amplitude of the wave electric field in the pulse peak; $\omega_{n}$ is the characteristic frequency of wave field oscillation; $k_{n}=\left(\omega_{n} / c, \mathbf{k}_{n}\right)$ are the wave vectors; $\delta_{n}$ are the wave ellipticity parameters $\left(\delta_{n}=0\right.$ corresponds to the linear polarization case, $\delta_{n}= \pm 1$ corresponds to the circular polarization case); $e_{n x, n y}=\left(0, \mathbf{e}_{n x, n y}\right)$ are the four-vectors of wave polarization, meeting the conditions:

$$
e_{n x, n y}^{2}=-1, \quad\left(e_{n x, n y} k_{n}\right)=k_{n}^{2}=0 .
$$

The functions $g_{n}\left(\varphi_{n} / \omega_{n} \tau_{n}\right)$ in (1) are the envelope functions of the four-potential of external wave that allows to take into account the pulsed character of the laser field. Generally the envelope function is chosen to be equal to unity in the center of a pulse and to decrease exponentially when $\left|\varphi_{n}\right| \gg \omega_{n} \tau_{n}$. Thereby the quantity $\tau_{n}$ can be considered as the characteristic pulse duration [14]. It is assumed that pulse duration considerably exceeds the characteristic period of wave oscillation (see, (5)). The process is studied in the frame of the quasimonochromatic approximation when each of the waves performs a lot of amplitude oscillation, i.e. the fallowing condition is met:

$$
\omega_{n} \tau_{n} \gg 1 \text {. }
$$

The quantity $\omega_{n} \tau_{n} / 2 \pi$ is the characteristic number of oscillation of the external field amplitude in the electromagnetic pulse. The given condition (5) allows to apply the derived differential cross-section for the real experiment description. It is shown in [14], that in addition to fulfillment of the condition of ponderomotive scattering neglecting, which is caused by spatial inhomogeneity of the laser field amplitude, the pulse duration must to be small enough that spreading of the initial particles packet can be neglected. Within the optical frequency range $\tau_{n}$ can be even tens of femtoseconds. Consequently, the condition (5) is rather weak and is satisfied for the majority of modern lasers. Such fields, for which the condition (5) is met, are named the quasimonochromatic [6-8,14]. We emphasize here that field description by the potential (1)-(3) doesn't take into account the possible shift between laser waves and stipulates that laser pulses' maxima coincide. We do not study the effects introduced by the phase shift and dispersion in the pulses' peaks in the presented paper.

Note that potential envelopes $g_{n}\left(\varphi_{n} / \omega_{n} \tau_{n}\right)$ are chosen as functions of variables $\varphi_{n}$, therefore an electromagnetic field with the four-potential (1)-(3) represents a plane wave. Thereby exact solutions of the Dirac equation for an electron in the field of a plane wave of an arbitrary spectral composition (the Volkov functions) can be used for description of the electron state in the field of a quasimonochromatic wave $[6-8,14,45-56]$.

Nonlinear effects in processes of interaction of electrons with the wave field and with each other are specified by the following parameters. The first one is the relativistically invariant classical parameter [2] 


$$
\eta_{0 n}^{(j)}=\frac{e F_{0 n}}{m c \omega_{n}}, \quad j=1,2,
$$

which numerically equals to the ratio of the work done by the field within the wavelength to the electron rest energy (here, $e$ is the electron charge and $m$ is the electron mass, the index $j$ labels the particle). The parameter (6) is one the external-field most important characteristics, and if $\eta_{0 n}^{(j)} \ll 1$ it has the meaning of the velocity of particle oscillation in the external laser field.

The multiplicity of multiphoton processes occurring in Coulomb interaction of particles in the laser field in nonresonant scattering are determined by the quantum Bunkin-Fedorov multiphoton parameter [56]

$$
\gamma_{0 n}^{(j)} \sim \eta_{0 n}^{(j)} \frac{m v_{j} c}{\hbar \omega_{n}},
$$

which numerically equals to the ratio of the work done by the field within the space, passing by an electron during the characteristic duration of wave oscillation $\left(\omega_{n}^{-1}\right)$, to the wave photon energy (here, $\hbar$ is the Planck constant) (the exact expression for the parameter $\gamma_{0 n}^{(j)}$ in electron-electron scattering is given by (33)).

In the relativistic case and for laser wave elliptical polarization the quantum parameter $y_{0 n}^{(j)}$ appears [5]

$$
y_{0 n}^{(j)}=\left(1-\delta_{n}^{2}\right)\left(\frac{m c^{2}}{E_{j}}\right) \eta_{0 n}^{(j)} \gamma_{0 n}^{(j)},
$$

which equals zero for wave circular polarization and in the dipole approximation with respect to interaction of an electron with the wave field. The parameters $\gamma_{0 n}^{(j)}, y_{0 n}^{(j)}$ determine stimulated emission and absorption of photons of the first and second wave by independently of one another.

As previously mentioned the special quite narrow region named the interference one appears in the field of two laser waves, where the Bunkin-Fedorov quantum parameter doesn't appear. When processes in the laser field of two unidirectional waves of different frequency are studied the quantum interference parameter may be relevant $[5,24]$ :

$$
\alpha_{0 \pm}^{(j)}=\eta_{01}^{(j)} \eta_{02}^{(j)} \frac{\left|\mathbf{p}_{j}\right| \cdot c}{\hbar\left(\omega_{1} \pm \omega_{2}\right)}\left(\frac{m c^{2}}{E_{j}}\right)^{2} .
$$

Parameters $\alpha_{0 \pm}^{(j)}(9)$ determine interference processes in correlated stimulated emission and absorption of photons of both waves by electrons. Here, the upper sign corresponds to the waves' different polarization case: $\delta_{1}=-\delta_{2}=+1$, and the lower sign - to the same polarization case: $\delta_{1}=\delta_{2}=+1$. Also we emphasize that in our case the frequency of waves should not be close for waves' different polarization, because it corresponds to the single wave case (see, [24]).

For a certain intensity of the external field the parameters (6), (7), (9) assume substantially different values. For example, in the scattering region, where the interference effect doesn't reveal, for the optical frequency $\left(\omega_{n} \sim 10^{15} c^{-1}\right)$ the classical parameter $\eta_{0 n}^{(j)} \sim 1$ in the field with the strength $F_{0 n} \sim 10^{10} \div 10^{11} \mathrm{~V} / \mathrm{cm}$; the quantum Bunkin-Fedorov parameter $\gamma_{0 n}^{(j)} \sim 1$ for the field strength $F_{0 n} \sim\left(10^{5} \div 10^{6}\right)\left(c / v_{j}\right) \mathrm{V} / \mathrm{cm}$. The quantum interference parameter $\alpha_{0 \pm}^{(j)} \sim 1$ for the field strength $F_{0 n} \sim 10^{7} \div 10^{8} \mathrm{~V} / \mathrm{cm}$ (for nonrelativistic electrons, if $\left.v_{j} \sim 0.1\right)$. For the optical frequency, where in the interference region the parameters $\alpha_{0 \pm}^{(j)} \sim 1$, out of the interference region the parameter $\gamma_{0 n}^{(j)} \sim 10^{3}$. This implies that in the optical frequency $\gamma_{0 n}^{(j)} \gg \eta_{0 n}^{(j)}$. Thereby such problems are usually considered in the moderately strong field, for which the parameters $\eta_{0 n}^{(j)}$ and $\gamma_{0 n}^{(j)}$ satisfy the following conditions

$$
\begin{gathered}
\eta_{0 n}^{(j)} \sim 1, \quad\left(\eta_{0 n}^{(j)}\right)^{2} \ll 1, \\
\gamma_{0 n}^{(j)} \gtrsim 1,
\end{gathered}
$$

and for the interference region we have

$$
\alpha_{0 \pm}^{(j)} \gtrsim 1
$$

In (10) the values of the classical parameter $\eta_{0 n}^{(j)} \lesssim 0.3$ are mentioned [10].

It is important to underline that for nonresonant scattering of an electron by an electron in the field of the both single wave and two waves the Bunkin-Fedorov quantum parameter $\gamma_{0 n}^{(j)}(7)$ is the main multiphoton parameter [5-8]. In resonant scattering of an electron by an electron the influence of the parameter $\gamma_{0 n}^{(j)}$ doesn't reveal (this parameter becomes a classical one and takes the value of the order of the parameter $\eta_{0 n}^{(j)}$ due to the resonant conditions). In this case the classical parameter $\eta_{0 n}^{(j)}$ is the main multiphoton parameter [45]. At that in the field of two waves in the interference resonant region the quantum parameter $\alpha_{0 \pm}^{(j)}$ (9) also becomes classical (see, (54)). Therefore hereinafter we consider resonant scattering of an electron by an electron in the intensity range (10), i.e. in the first order of the perturbation theory with respect to the laser field.

The relativistic system of units, where $\hbar=c=1$, and the standard metric for four-vectors: $(a b)=a_{0} b_{0}-\mathbf{a b}$ will be used throughout this paper.

\section{Process General Amplitude}

We limit to the Feynman direct diagram only when calculating the amplitude (Figure 1) $[19,41,45]$. Such a problem statement is possible due to fact that the resonances 
for the direct diagrams of scattering type and the ones for the exchange diagrams within the intensity range (10) occur within the considerably different nonoverlapping kinematical regions [41,45]. Note also that for the direct scattering amplitude within the fields range (10) resonant scattering of an electron by an electron occurs when electrons scatter forwards into the small angles in the frame of the reference related to the center of inertia of initial particles [41,45] and is effectively divided into two processes of the first order similar to the Compton scattering of a wave by an electron (laser-induced Compton-effect).

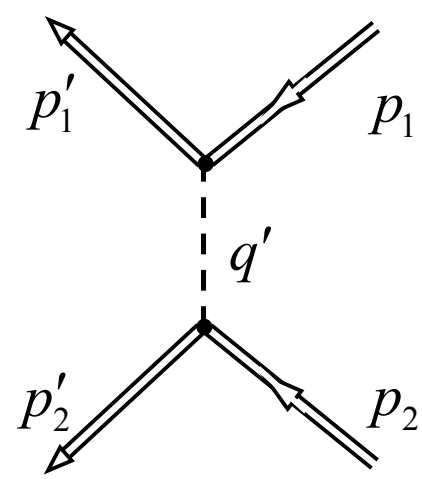

Figure 1. The Feynman direct diagram of scattering of an electron by an electron in the field of two pulsed laser waves. The external incoming and outgoing double lines correspond to the wave functions of electrons in initial and final states in the field of two plane pulsed waves (the Volkov functions), and the inner dashed line corresponds to a Green function of a free photon.

The general expression for the process scattering amplitude (for the direct diagram) has the form [18]:

$$
\begin{aligned}
& S=i e^{2} \int d^{4} x_{1} d^{4} x_{2} D_{v v^{\prime}}\left(x_{1}-x_{2}\right) \times \\
& {\left[\bar{\psi}_{p_{1}^{\prime}}\left(x_{1} \mid A\right) \tilde{\gamma}^{v} \psi_{p_{1}}\left(x_{1} \mid A\right)\right]\left[\bar{\psi}_{p_{2}^{\prime}}\left(x_{2} \mid A\right) \tilde{\gamma}^{v^{\prime}} \psi_{p_{2}}\left(x_{2} \mid A\right)\right] .}
\end{aligned}
$$

Here, $\tilde{\gamma}^{v}$ and $\tilde{\gamma}^{v^{\prime}}\left(v, v^{\prime}=0,1,2,3\right)$ are the Dirac matrices (summation over indices $v, v^{\prime}$ in (13) is expected); $D_{v v^{\prime}}\left(x_{1}-x_{2}\right)$ is the Green function of an intermediate free photon; $\psi_{p_{j}}(x \mid A)$ and $\bar{\psi}_{p_{j}^{\prime}}(x \mid A)$ are the wave functions of initial and final electrons in the field of two pulsed laser waves, respectively:

$$
\begin{gathered}
\psi_{p_{j}}\left(x_{j} \mid A\right)=\left[1+\frac{e}{2\left(n_{k} p_{j}\right)} \hat{n}_{k} \hat{A}\right] \frac{u_{j}}{\sqrt{2 E_{j}}} \exp \left(i S_{j}\right), \\
\bar{\psi}_{p_{j}^{\prime}}\left(x_{j} \mid A\right)=\frac{\bar{u}_{j}^{\prime}}{\sqrt{2 E_{j}^{\prime}}}\left[1+\frac{e}{2\left(n_{k} p_{j}^{\prime}\right)} \hat{A} \hat{n}_{k}\right] \exp \left(i S_{j}^{\prime}\right), \\
S_{j}=-\left(p_{j} x\right)-\int_{-\infty}^{\varphi} \frac{e}{\left(k p_{j}\right)}\left[\left(p_{j} A\right)-\frac{e}{2} A^{2}\right] d \varphi .
\end{gathered}
$$

Here, $n_{k}=k_{n} / \omega_{n} ; u_{j}, \bar{u}_{j}^{\prime}$ are the Dirac bispinors for electron initial and final states, respectively; $p_{j}=\left(E_{j}, \mathbf{p}_{j}\right)$ and $p_{j}^{\prime}=\left(E_{j}^{\prime}, \mathbf{p}_{j}^{\prime}\right)$ are particle four-momenta. Hereafter expressions with hats above mean the scalar product of the corresponding four-vector with the Dirac matrices $\left(\hat{k}=k_{v} \tilde{\gamma}^{v}=k_{0} \tilde{\gamma}_{0}-\mathbf{k} \tilde{\gamma}\right)$

After computations analogous to the single pulsed wave case (see, $[22,41,45])$ the direct amplitude of scattering of an electron with the four-momentum $p_{1}$ by an electron with the four-momentum $p_{2}$ in the field of two pulsed moderately strong waves (5), (10) with the four-potential (1)-(3) can be represented as a sum over partial components with emission $\left(l_{1}^{(1)}>0, l_{2}^{(1)}>0, l_{1}^{(2)}>0, l_{2}^{(2)}>0\right)$ and absorption $\left(l_{1}^{(1)}<0, l_{2}^{(1)}<0, l_{1}^{(2)}<0, l_{2}^{(2)}<0\right)$ of external field photons:

$$
S_{f i}=\sum_{l_{1}^{(1)}=-\infty}^{\infty} \sum_{l_{2}^{(1)}=-\infty}^{\infty} \sum_{l_{1}^{(2)}=-\infty}^{\infty} \sum_{l_{2}^{(2)}=-\infty}^{\infty} S^{\left(l_{1}^{(1)} l_{2}^{(1)} l_{1}^{(2)} l_{2}^{(2)}\right)}
$$

where $l_{n}^{(j)}$ is the number of $n$-wave's photons emitted or absorbed by an $j$-particle.

$$
S^{\left(l_{1}^{(1)} l_{2}^{(1)} l_{1}^{(2)} l_{2}^{(2)}\right)}=\frac{i e^{2}(2 \pi)^{3} \omega_{1} \tau_{1}^{2}}{8 \sqrt{E_{1} E_{2} E_{1}^{\prime} E_{2}^{\prime}}} \delta^{2}\left(\mathbf{q}_{\perp}\right) \delta\left(q_{-}\right) D_{l_{1}^{(1)} l_{2}^{(1)} l_{1}^{(2)} l_{2}^{(2)}} .
$$

Here, the arguments of delta-functions are defined by the components of the four-vector $q=\left(q_{0}, \mathbf{q}\right)$

$$
\begin{gathered}
q=p_{1}+p_{2}-p_{1}^{\prime}-p_{2}^{\prime}+\left(l_{1}^{(1)}+l_{1}^{(2)}\right) k_{1}+\left(l_{2}^{(1)}+l_{2}^{(2)}\right) k_{2}, \\
\left\{\begin{array}{l}
\mathbf{q}_{\perp}=\left(q_{x}, q_{y}\right), \\
q_{-}=q_{0}-q_{z}, \\
q_{+}=\left(q_{0}+q_{z}\right) / 2 ;
\end{array}\right. \\
D_{l_{1}^{(1)} l_{2}^{(1)} l_{1}^{(2)} l_{2}^{(2)}}=\int_{-\infty}^{\infty} \frac{\left(\vec{u}_{1}^{\prime} \Lambda_{l_{1}^{(1)} l_{2}^{(1)}}^{v}(\zeta) u_{1}\right)\left(\bar{u}_{2}^{\prime} \Lambda_{l_{1}^{(2)} l_{2}^{(2)}, v}(\zeta) u_{2}\right)}{q^{\prime 2}+2 \zeta\left(k_{1} q^{\prime}\right)+i 0} d \zeta .
\end{gathered}
$$

In the expression (21) $q^{\prime}$ is the four-momentum of an intermediate virtual photon:

$$
q^{\prime}=p_{1}-p_{1}^{\prime}+l_{1}^{(1)} k_{1}+l_{2}^{(1)} k_{2}=p_{2}^{\prime}-p_{2}-l_{1}^{(2)} k_{1}-l_{2}^{(2)} k_{2},
$$

and the functions $\Lambda_{l_{1}^{(1)} l_{2}^{(1)}}^{v}(\zeta), \Lambda_{l_{1}^{(2)} l_{2}^{(2)}, v}(\zeta)$ have the form:

$$
\left\{\begin{array}{l}
\Lambda_{l_{1}^{(1)} l_{2}^{(1)}}^{v}(\zeta)=\int_{-\infty}^{\infty} d \phi_{1} G_{l_{1}^{(1)} l_{2}^{(1)}}^{v}\left(\phi_{1}\right) \exp \left\{i \omega_{1} \tau_{1} \zeta \phi_{1}\right\}, \\
\Lambda_{l_{1}^{(2)} l_{2}^{(2)}, v}(\zeta)=\int_{-\infty}^{\infty} d \phi_{2} G_{l_{1}^{(2)} l_{2}^{(2)}, v}\left(\phi_{2}\right) \exp \left\{i\left(q_{+} \tau_{1}-\omega_{1} \tau_{1} \zeta\right) \phi_{2}\right\} .
\end{array}\right.
$$

The integration variables $\phi_{n}$ have the form

$$
\phi_{n}=\frac{\varphi_{n}}{\omega_{n} \tau_{n}},
$$

(the expression for $\varphi_{n}$ is given by (2)). 
Functions $G_{l_{1}^{(1)} l_{2}^{(1)}}^{v}\left(\phi_{1}\right), G_{l_{1}^{(2)} l_{2}^{(2)}, v}\left(\phi_{2}\right)$ in (23) are defined as

$$
\begin{gathered}
G_{l_{1}^{(1)} l_{2}^{(1)}}^{v}\left(\phi_{1}\right)=\tilde{\gamma}^{v} I_{l_{1}^{(1)} l_{2}^{(1)}}\left(\phi_{1}\right)+\frac{m}{4 \kappa_{1}^{\prime}} \tilde{\gamma}^{v} \hat{n}_{k} \hat{D}_{l_{1}^{(1)} l_{2}^{(1)}}\left(\phi_{1}\right)+ \\
+\frac{m}{4 \kappa_{1}} \hat{D}_{l_{1}^{(1)} l_{2}^{(1)}}\left(\phi_{1}\right) \hat{n}_{k} \tilde{\gamma}^{v}, \\
\hat{D}_{l_{1}^{(1)} l_{2}^{(1)}}\left(\phi_{1}\right)=\eta_{01}^{(1)} g_{1}\left(\phi_{1}\right)\left[\hat{\varepsilon}_{1} I_{l_{1}^{(1)}+1, l_{2}^{(1)}}\left(\phi_{1}\right)+\hat{\varepsilon}_{1}^{*} I_{l_{1}^{(1)}-1, l_{2}^{\left(l^{1}\right)}}\left(\phi_{1}\right)\right]+ \\
+\eta_{02}^{(1)} g_{2}\left(\frac{\tau_{1}}{\tau_{2}} \phi_{1}\right)\left[\hat{\varepsilon}_{2} I_{l_{1}^{(1)}, l_{2}^{(1)}+1}\left(\phi_{1}\right)+\hat{\varepsilon}_{2}^{*} I_{l_{1}^{(1)}, l_{2}^{(1)}-1}\left(\phi_{1}\right)\right], \\
G_{l_{1}^{(2)} l_{2}^{(2)}, v}\left(\phi_{2}\right)=\tilde{\gamma}_{v} I_{l_{1}^{(2)} l_{2}^{(2)}}\left(\phi_{2}\right)+\frac{m}{4 \kappa_{2}^{\prime}} \tilde{\gamma}_{v} \hat{n}_{k} \hat{D}_{l_{1}^{(2)} l_{2}^{(2)}}\left(\phi_{2}\right)+ \\
\frac{m}{4 \kappa_{2}} \hat{D}_{l_{1}^{(2)} l_{2}^{(2)}}\left(\phi_{2}\right) \hat{n}_{k} \tilde{\gamma}_{v}, \\
\hat{D}_{l_{1}^{(2)} l_{2}^{(2)}}\left(\phi_{2}\right)=\eta_{01}^{(2)} g_{1}\left(\phi_{2}\right)\left[\hat{\varepsilon}_{1} I_{l_{1}^{(2)}+1, l_{2}^{(2)}}\left(\phi_{2}\right)+\hat{\varepsilon}_{1}^{*} I_{l_{1}^{(2)}-1, l_{2}^{\left(l^{2}\right)}}\left(\phi_{2}\right)\right]+ \\
+\eta_{02}^{(2)} g_{2}\left(\frac{\tau_{1}}{\tau_{2}} \phi_{2}\right)\left[\hat{\varepsilon}_{2} I_{l_{1}^{(2)}, l_{2}^{(2)}+1}\left(\phi_{2}\right)+\hat{\varepsilon}_{2}^{*} I_{l_{1}^{(2)}, l_{2}^{(2)}-1}\left(\phi_{2}\right)\right], \\
\varepsilon_{n}=e_{n x}+i \delta_{n} e_{n y} .
\end{gathered}
$$

Through $\kappa_{j}$ and $\kappa_{j}^{\prime}$ the following relations are designated

$$
\left\{\begin{array}{l}
\kappa_{j}=E_{j}-\mathbf{e}_{z} \mathbf{p}_{j}, \\
\kappa_{j}^{\prime}=E_{j}^{\prime}-\mathbf{e}_{z} \mathbf{p}_{j}^{\prime},
\end{array}\right.
$$

where $\mathbf{e}_{z}$ is the unit vector along the direction of external waves propagating $\mathbf{e}_{z}=\mathbf{k}_{n} /\left|\mathbf{k}_{n}\right|$.

In expressions for $G_{l_{1}^{(1)} l_{2}^{(1)}}^{v}\left(\phi_{1}\right)$ (25) and $G_{l_{1}^{(2)} l_{2}^{(2)}, v}\left(\phi_{2}\right)$

functions $I_{l_{1}^{(1)} l_{2}^{(1)}}, I_{l_{1}^{(2)} l_{2}^{(2)}}$ determine multiphoton processes in the field of two laser waves. These functions are studied in detail in $[5,57]$ and have the following form

$$
\begin{aligned}
& I_{r_{1} r_{2}}\left(\phi_{n}\right) \equiv I_{r_{1} r_{2}}\left(\chi_{n}^{(j)}, \gamma_{n}^{(j)}, y_{n}^{(j)} ; \alpha_{+}^{(j)}, \alpha_{-}^{(j)}, \tau_{+}, \tau_{-}\right)= \\
& =\frac{1}{(2 \pi)^{2}} \int_{0}^{2 \pi} d \varphi_{1}^{\prime} \int_{0}^{2 \pi} d \varphi_{2}^{\prime} \times \\
& \exp \left\{\sum_{n=1}^{2}\left(i \gamma_{n}^{(j)} \sin \left(\varphi_{n}^{\prime}-\chi_{n}^{(j)}\right)+i y_{n}^{(j)} \sin 2 \varphi_{n}^{\prime}-i r_{n} \varphi_{n}^{\prime}\right)\right\} \times \\
& \exp \left\{i \alpha_{+}^{(j)} \sin \left(\varphi_{1}^{\prime}+\varphi_{2}^{\prime}-\tau_{-}\right)+i \alpha_{-}^{(j)} \sin \left(\varphi_{1}^{\prime}-\varphi_{2}^{\prime}-\tau_{+}\right)\right\},
\end{aligned}
$$

with the arguments

$$
\begin{gathered}
\gamma_{n}^{(j)} \equiv \gamma_{0 n}^{(j)} \cdot g_{n}\left(\phi_{n}\right), \\
\gamma_{0 n}^{(j)}=\eta_{0 n}^{(j)} \frac{m}{\omega_{n}} \sqrt{\left(e_{n x} \tilde{g}_{j}\right)^{2}+\delta_{n}^{2}\left(e_{n y} \tilde{g}_{j}\right)^{2}},
\end{gathered}
$$

$$
\begin{gathered}
\tan \chi_{n}^{(j)}=\delta_{n} \frac{\left(e_{n y} \tilde{g}_{j}\right)}{\left(e_{n x} \tilde{g}_{j}\right)}, \\
\tilde{g}_{j}=\frac{p_{j}}{\kappa_{j}}-\frac{p_{j}^{\prime}}{\kappa_{j}^{\prime}}, \\
y_{n}^{(j)} \equiv y_{0 n}^{(j)} \cdot g_{n}^{2}\left(\phi_{n}\right), \\
y_{0 n}^{(j)}=\frac{1}{8}\left(1-\delta_{n}^{2}\right) m^{2} \frac{\left(\eta_{0 n}^{(j)}\right)^{2}}{\omega_{n}}\left(\frac{1}{\kappa_{j}}-\frac{1}{\kappa_{j}^{\prime}}\right), \\
\alpha_{ \pm}^{(j)} \equiv g_{1}\left(\phi_{n}\right) g_{2}\left(\phi_{n}\right) \alpha_{0 \pm}^{(j)}, \\
\alpha_{0 \pm}^{(j)}=\frac{m^{2} \eta_{01}^{(j)} \eta_{02}^{(j)}}{2\left(\omega_{1} \pm \omega_{2}\right)}\left|d_{\mp}\right|\left(\frac{1}{\kappa_{j}}-\frac{1}{\kappa_{j}^{\prime}}\right), \\
d_{ \pm}=\left(1 \pm \delta_{1} \delta_{2}\right) \cos \Delta+i\left(\delta_{1} \pm \delta_{2}\right) \sin \Delta, \\
\tau_{ \pm}=\frac{\operatorname{Im}\left(d_{ \pm}\right)}{\operatorname{Re}\left(d_{ \pm}\right)}=\frac{\delta_{1} \pm \delta_{2}}{1 \pm \delta_{1} \delta_{2}} \tan \Delta,
\end{gathered}
$$

where the angle $\Delta$ defines relative position of vectors of field strength of laser waves.

The expression (31) gives the integral representation for the function $I_{r_{1} r_{2}}$. These functions also can be represented in the form of expansion in series of Bessel functions [57]. They determine the probability of virtual correlated emission and absorption of external-wave photons by initial and final electrons, and the intensity of such virtual processes is determined by parameters $\alpha_{0 \pm}^{(j)}$ :

$$
\begin{aligned}
& I_{r r^{\prime}}=\sum_{s_{1}=-\infty}^{\infty} \sum_{s_{2}=-\infty}^{\infty} \exp \left\{-i\left(s_{1} \tau_{-}+s_{2} \tau_{+}\right)\right\} \times \\
& J_{s_{1}}\left(\alpha_{+}^{(j)}\right) J_{s_{2}}\left(\alpha_{-}^{(j)}\right) \times \\
& L_{r-s_{1}-s_{2}}\left(\chi_{1}^{(j)}, \gamma_{1}^{(j)}, y_{1}^{(j)}\right) L_{r^{\prime}-s_{1}+s_{2}}\left(\chi_{2}^{(j)}, \gamma_{2}^{(j)}, y_{2}^{(j)}\right) .
\end{aligned}
$$

Here, $J_{s_{1}, s_{2}}$ are integer-order Bessel functions. The special functions $L_{r}\left(\chi_{n}^{(j)}, \gamma_{n}^{(j)}, y_{n}^{(j)}\right)$ are determined by parameters of the first and second laser wave [57]. They also can be represented as expansion in series of Bessel functions

$$
\begin{aligned}
& L_{r}\left(\chi_{n}^{(j)}, \gamma_{n}^{(j)}, y_{n}^{(j)}\right)= \\
& \exp \left(-i r \chi_{n}\right) \sum_{s=-\infty}^{\infty} \exp \left(2 i s \chi_{n}^{(j)}\right) J_{r-2 s}\left(\gamma_{n}^{(j)}\right) J_{s}\left(y_{n}^{(j)}\right) .
\end{aligned}
$$

For the interference region (see, (46)) and wave circular polarization $\left(\delta_{n}^{2}=1\right)$ the expression for the special function(42) simplifies considerably [5,57]

$$
I_{r_{1} r_{2}} \rightarrow I_{r_{ \pm}}=\exp \left(i r_{ \pm} \chi_{ \pm}\right) J_{r_{ \pm}}\left(\alpha_{ \pm}^{(j)}\right), r_{ \pm}=\left(r_{1} \pm r_{2}\right) / 2,
$$

where the upper sign corresponds to the waves' different 
polarization case: $\delta_{1}=-\delta_{2}=+1$, and the lower sign - to the same polarization case: $\delta_{1}=\delta_{2}=+1$. Integer indices $r_{+}, r_{-}$ $\left(r_{ \pm}=0, \pm 1, \pm 2, \ldots\right)$ determine the number of photons of combination frequency

$$
\Omega_{ \pm}=\omega_{1} \pm \omega_{2}
$$

emitted or absorbed by electrons.

One can see from (44) that in the interference kinematical region for circular polarization of both waves the scattering process formally looks like the field of a single wave, but with emission and absorption of photons of combination frequency $\Omega_{ \pm}$(45).

Hereinafter we consider $\omega_{1}>\omega_{2}$. Note also that if waves' frequency and polarization are close to each other, then it is easy to demonstrate that the obtained amplitude (17)-(28) is transformed into the amplitude for the single laser wave case [24].

\section{Interference Resonant Amplitude}

It is of interest to define and study the interference kinematical region, where parameters $\gamma_{0 n}^{(j)}$ are small due to the special geometry of the considered process in the field of two waves. This region is determined by the following condition:

$$
\gamma_{0 n}^{(j)}=0
$$

Parameters $\gamma_{0 n}^{(j)}$ can be represented in the form:

$$
\begin{gathered}
\gamma_{0 n}^{(j)}=\eta_{0 n}^{(j)}\left|\tilde{\boldsymbol{g}}_{j}^{\|}\right| \sqrt{\cos ^{2} \psi_{n}+\delta_{n}^{2} \sin ^{2} \psi_{n}}, \\
\psi_{n}^{(j)}=\angle\left(\tilde{\boldsymbol{g}}_{j}^{\|}, \mathbf{e}_{n x}\right),
\end{gathered}
$$

where $\tilde{\boldsymbol{g}}_{j}^{\|}$is the projection of the vector $\tilde{\boldsymbol{g}}_{j}$ onto the plane, perpendicular to $\mathbf{e}_{z}$. From the expression (47) we can conclude that it is possible for both field arbitrary intensity and polarization in the kinematical region if the vector $\tilde{\boldsymbol{g}}_{j}^{\|}$is parallel or antiparallel to the $z$-axis (i.e., is perpendicular to the polarization plane):

$$
\left(\mathbf{e}_{n x} \tilde{\boldsymbol{g}}_{j}\right)=\left(\mathbf{e}_{n y} \tilde{\boldsymbol{g}}_{j}\right)=0 \Rightarrow \tilde{\boldsymbol{g}}_{j} \uparrow \uparrow \mathbf{e}_{z} \text { or } \tilde{\boldsymbol{g}}_{j} \uparrow \downarrow \mathbf{e}_{z} .
$$

These conditions obviously can be met only if electrons scatter in the plane formed by their initial momenta and the wave vector [24].

It was mentioned above that scattering of an electron by an electron in the laser-wave field can proceed resonantly. We consider the case when an intermediate photon falls within the mass shell. It means that the squared four-momentum of intermediate photon belongs to a very narrow region near zero. Consider the field pulsed character in the frame of quasimonochromatic wave (5) the condition of Oleinik resonance has the form:

$$
q^{\prime 2} \lesssim \frac{\left(k_{n} q^{\prime}\right)}{\omega_{n} \tau_{n}} .
$$

This region depends on initial momenta of both scattered particles and their scattering angles. Four-momentum conservation laws for the vertices of the resonant diagram (Figure 2) can be represented in the form of two equalities:

$$
\begin{array}{r}
p_{1}+l^{(1)}\left(k_{1} \pm k_{2}\right)=p_{1}^{\prime}+q^{\prime}, \quad l^{(1)} \geq 1, \\
p_{2}+q^{\prime}=p_{2}^{\prime}-l^{(2)}\left(k_{1} \pm k_{2}\right), \quad l^{(2)} \leq-1 .
\end{array}
$$

Here, the following condition was used (see, (22) and [24]):

$$
l_{2}^{(j)}= \pm l_{2}^{(j)}, \quad l^{(j)}=l_{1}^{(j)}, \quad j=1,2 .
$$

The equality (50) expresses the four-momentum conservation law in the process of emission of intermediate real photon by the first electron at the expense of absorption of $l^{(1)}$ combination photons $\Omega_{ \pm}$. The second equality (51) corresponds to the four-momentum conservation law in the process of absorption of intermediate real photon by the second electron with emission of $l^{(2)}$ combination photons $\Omega_{ \pm}$.
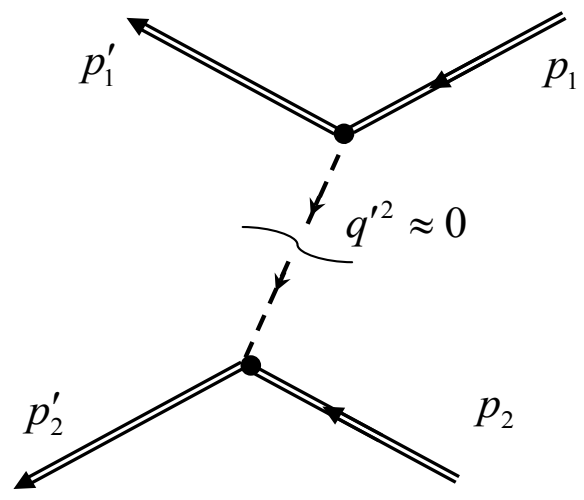

Figure 2. The Feynman diagram of resonant scattering of an electron by an electron in the field of two pulsed laser waves.

It is easy to demonstrate that for waves' circular polarization under the resonance condition (49) within the interference region the Bessel function arguments $\alpha_{0 \pm}^{(j)}$ become classical (under the resonance condition the Planck's constant in (33) is reduced) and can be represented in the following form

$$
\alpha_{0 \pm}^{(j)}=\frac{r_{ \pm}}{2} \eta_{01}^{(j)} \eta_{02}^{(j)}\left[\varsigma+\varsigma^{\prime}-1\right] \sim \eta_{01}^{(j)} \eta_{02}^{(j)} \ll 1
$$

Here,

$$
\varsigma \equiv\left(k_{n} q^{\prime}\right) /\left(k_{n} p_{j}^{\prime}\right), \quad \varsigma^{\prime} \equiv\left(k_{n} p_{j}^{\prime}\right) /\left(k_{n} p_{j}\right)
$$

are the relativistically invariant parameters.

We choose the potential envelope functions in the Gaussian form 


$$
g_{n}\left(\frac{\varphi_{n}}{\omega_{n} \tau_{n}}\right)=\exp \left\{-\left(\frac{2 \varphi_{n}}{\omega_{n} \tau_{n}}\right)^{2}\right\}=\exp \left\{-\left(2 \phi_{n}\right)^{2}\right\},
$$

and also use the frame of reference related to the center of inertia of initial particles, where $\mathbf{p}_{1}+\mathbf{p}_{2}=0$. At that initial particle energy have the form

$$
E_{i}=E_{1}=E_{2}=\sqrt{m^{2}+\mathbf{p}^{2}}, \mathbf{p}=\mathbf{p}_{1}=-\mathbf{p}_{2} .
$$

Final particle momenta and energy are determined as

$$
\begin{gathered}
\mathbf{p}_{1}^{\prime}=\frac{1}{2} \mathbf{P}^{\prime}+\mathbf{p}^{\prime}, \quad \mathbf{p}_{2}^{\prime}=\frac{1}{2} \mathbf{P}^{\prime}-\mathbf{p}^{\prime}, \\
E_{1}^{\prime}=\sqrt{m^{2}+\left(\frac{1}{2} \mathbf{P}^{\prime}+\mathbf{p}^{\prime}\right)^{2}} \approx \sqrt{m^{2}+\mathbf{p}^{\prime 2}}=E_{f}, \\
E_{2}^{\prime}=\sqrt{m^{2}+\left(\frac{1}{2} \mathbf{P}^{\prime}-\mathbf{p}^{\prime}\right)^{2}} \approx \sqrt{m^{2}+\mathbf{p}^{\prime 2}}=E_{f},
\end{gathered}
$$

where $\mathbf{P}^{\prime}$ is the momentum of the inertia center after scattering.

The scattering angle of electrons in the frame of reference related to the center of inertia can be determined as

$$
\theta=\angle\left(\mathbf{p}, \mathbf{p}^{\prime}\right)
$$

If take (49), (10) into account it is easy to conclude that for chosen direction of intermediate photon propagation (see, Figure 2) resonance appears onto small scattering angles $[5,41,45]$ :

$$
\theta_{n}^{r e s}=2 \frac{\omega_{n}}{|\mathbf{p}|} \sin \theta_{i} \sim \frac{\omega_{n}}{|\mathbf{p}|} \ll 1,
$$

where $\theta_{i}=\angle\left(\mathbf{e}_{z}, \mathbf{p}\right)$ is the angle between directions of wave propagation and initial relative momentum. Note that for the process direct amplitude there two angles which correspond to the first and second wave.

Thereby the resonant region of electron scattering angles in the frame of reference related to the center of inertia is the following:

$$
\left|\theta-\theta_{n}^{r e s}\right| \lesssim \frac{\theta_{n}^{\text {res }}}{\omega_{1} \tau_{1}} \ll \theta_{n}^{\text {res }}
$$

With that resonance for the exchange amplitude appears within considerably another kinematical region $[5,41,45]$. Using conditions (46), (49) we obtain that resonant scattering appears within the interference region if ingoing angle of the nonrelativistic electron satisfies the condition:

$$
\sin \theta_{i}=1-v_{j} \cos \theta_{i} .
$$

One can see that ingoing angles of nonrelativistic particles in the frame of reference related to the center of inertia has be close to $\pi / 2$ in the interference region:

$$
\theta_{i}=\frac{\pi}{2}-\tilde{\theta}, \quad \tilde{\theta} \cong 2 v_{j} \ll 1 .
$$

After escape into the interference region (see, [5]) we finally obtain the amplitude of resonant scattering of an electron by an electron in the field of two pulsed waves in the first order of the perturbation theory in the frame of reference related to the center of inertia in the form

$$
\begin{array}{r}
S^{r e s}=-\frac{e^{2}(2 \pi)^{4} m^{2}}{2^{5} \mathbf{p}^{2} E_{i} E_{f} \kappa_{1} \kappa_{2}} \delta^{2}\left(\mathbf{P}_{\perp}^{\prime}\right) \delta\left(E^{\prime}-E-P_{z}^{\prime}\right) \hat{M}, \\
\kappa_{1}=E_{1}-|\mathbf{p}| \cos \theta_{i}, \quad \kappa_{2}=E_{2}+|\mathbf{p}| \cos \theta_{i},
\end{array}
$$

where $E=2 E_{i}$ and $E^{\prime}=E_{1}^{\prime}+E_{2}^{\prime}$ are the particle total energy before and after scattering, respectively;

$$
\begin{aligned}
& M=\frac{\sqrt{\pi} \omega_{1} \tau_{1}^{2}}{\theta \cdot \theta_{n}^{r e s}} \int_{-\infty}^{\infty} d \phi \cdot \exp \left(i q_{+} \tau_{1} \phi\right) \times \\
& \left(\bar{u}_{1}^{\prime}\left\{c_{1}\left(\beta_{1}\right) \eta_{01}^{(1)}\left(n^{v} \hat{\varepsilon}_{1}-\varepsilon_{1}^{v} \hat{n}\right)+c_{2}\left(\beta_{2}\right) \eta_{02}^{(1)}\left(n^{v} \hat{\varepsilon}_{2}-\varepsilon_{2}^{v} \hat{n}\right)\right\} u_{1}\right) \times \\
& \left(\bar{u}_{2}^{\prime}\left\{b_{1} \eta_{01}^{(2)}\left(n_{\nu} \hat{\varepsilon}_{1}^{*}-\varepsilon_{1, v}^{*} \hat{n}\right)+b_{2} \eta_{02}^{(2)}\left(n_{\nu} \hat{\varepsilon}_{2}^{*}-\varepsilon_{2, \nu}^{*} \hat{n}\right)\right\} u_{2}\right) .
\end{aligned}
$$

The following designations are input in the function (66):

$$
\begin{gathered}
c_{1}\left(\beta_{1}\right)=e^{-\frac{\beta_{1}^{2}}{4}} e^{2 i \beta_{1} \phi}\left(\operatorname{erf}\left(2 \phi+i \frac{\beta_{1}}{2}\right)+1\right), \\
c_{2}\left(\beta_{2}\right)=\frac{\tau_{2}}{\tau_{1}} e^{-\frac{\beta_{2}^{2}}{4}} e^{2 i \beta_{2} \phi}\left(\operatorname{erf}\left(\frac{2 \tau_{2}}{\tau_{1}} \phi+i \frac{\beta_{2}}{2}\right)+1\right), \\
b_{1}=\exp \left(-(2 \phi)^{2}\right), \quad b_{2}=\exp \left(-\left(\frac{2 \tau_{1}}{\tau_{2}} \phi\right)^{2}\right), \\
q_{+}=\left(E^{\prime}-E+P_{z}^{\prime}\right) / 2 .
\end{gathered}
$$

The function "erf" in (67), (68) is the error function. The parameters $\beta_{1}$ (67) and $\beta_{2}$ (68) in (66) specify how the squared four-momentum of intermediate photon is close to the mass shell. These parameters are defined by both the process kinematics and characteristics of pulsed waves:

$$
\begin{aligned}
& \beta_{1} \equiv \frac{q_{1}^{\prime 2}}{4\left(k_{1} q_{1}^{\prime}\right)} \omega_{1} \tau_{1}, \quad \beta_{2} \equiv \frac{q_{2}^{\prime 2}}{4\left(k_{1} q_{2}^{\prime}\right)} \omega_{1} \tau_{1}, \\
& q_{1}^{\prime}=p_{1}-p_{1}^{\prime}+k_{1}=p_{2}^{\prime}-p_{2}+k_{1}, \\
& q_{2}^{\prime}=p_{1}-p_{1}^{\prime}+k_{2}=p_{2}^{\prime}-p_{2}+k_{2} .
\end{aligned}
$$

The delta-functions presence in the resonant amplitude (64) indicates fulfillment of the following conservation laws:

$$
\mathbf{P}_{\perp}^{\prime}=\left(P_{x}^{\prime}, P_{y}^{\prime}\right)=0, \quad E^{\prime}-E=P_{z}^{\prime},
$$

where $\mathbf{P}^{\prime}=\left(P_{x}^{\prime}, P_{y}^{\prime}, P_{z}^{\prime}\right)$ is the momentum of the inertia center after scattering.

\section{Resonant Cross Section}


We obtain the differential cross-section of resonant scattering of nonpolarized electron by an electron in the field of two pulsed laser wave into the element of solid angle within the interference region by a standard mode [18]

$$
\frac{d \sigma^{r e s}}{d \Omega^{\prime}}=\frac{(2 \pi)^{4} r_{e}^{2} m^{6}}{4 \mathrm{p}^{4} E_{i}^{2}} f^{r e s} .
$$

Here, $r_{e}$ is the classical electron radius. Resonant function $f^{\text {res }}$ consists of two terms, each of them corresponds to scattering of electrons onto one of resonant angles $\theta_{1}^{\text {res }}$ or $\theta_{2}^{\text {res }}$ :

$$
\begin{gathered}
f^{r e s}=f_{1}^{r e s}\left(\rho, \beta_{1}\right)+f_{2}^{r e s}\left(\rho, \beta_{2}\right), \\
f_{1}^{r e s}\left(\rho, \beta_{1}\right) \equiv\left(\eta_{01}^{(1)} \eta_{01}^{(2)}\right)^{2}\left(\frac{\omega_{1} \tau_{1}}{\theta \cdot \theta_{1}^{r e s}}\right)^{2} \cdot f_{1}\left(\rho, \beta_{1}\right), \\
f_{1}\left(\rho, \beta_{1}\right)=\frac{1}{\rho} \int_{0}^{\rho} d \phi\left|c_{1}\left(\beta_{1}\right)\right|^{2}\left\{b_{1}^{2}+k_{\delta} b_{2}^{2}\left(\frac{\eta_{02}^{(2)}}{\eta_{01}^{(1)}}\right)^{2}\right\}, \\
f_{2}^{\text {res }}\left(\rho, \beta_{2}\right) \equiv\left(\eta_{02}^{(1)} \eta_{02}^{(2)}\right)^{2}\left(\frac{\omega_{1} \tau_{1}}{\theta \cdot \theta_{2}^{\text {res }}}\right)^{2} \cdot f_{2}\left(\rho, \beta_{2}\right), \\
f_{2}\left(\rho, \beta_{2}\right)=\frac{1}{\rho} \int_{0}^{\rho} d \phi\left|c_{2}\left(\beta_{2}\right)\right|^{2}\left\{b_{2}^{2}+k_{\delta} b_{1}^{2}\left(\frac{\eta_{01}^{(2)}}{\eta_{02}^{(1)}}\right)^{2}\right\} \\
\rho=T / \tau_{1},
\end{gathered}
$$

where $\rho$ is the ratio of the observation time $T\left(T \gtrsim \tau_{1,2}\right)$ and the characteristic duration of a laser pulse (the first wave characteristic duration $\tau_{1}$ is chosen for concretization). This parameter acquires its physical meaning for the concrete conditions of the process. Thus, if the external field represents itself a series of consecutive laser pulses, then the parameter $\rho$ is the ratio of the time between peaks of adjacent pulses and the characteristic duration of a single laser pulse. Expressions for $c_{1}\left(\beta_{1}\right), c_{2}\left(\beta_{2}\right), b_{1}, b_{2}$ are given by (67)-(69).

Note also that the first term under the integral sign in the function $f_{1}$ (76) specifies emission and absorption of photons of the first wave, the second term specifies absorption of a photon from the first wave with emission of a photon of the second wave and quite the contrary for the function $f_{2}$.

The coefficient $k_{\delta}$ depends on wave polarization:

$$
k_{\delta}=\frac{1}{4}\left[\left(1+\delta_{1} \delta_{2}\right)^{2} \cos ^{2} \Delta+\left(\delta_{1}+\delta_{2}\right)^{2} \sin ^{2} \Delta\right] .
$$

One can notice from the expression (80) that in considered case of wave circular polarization

$$
\begin{cases}k_{\delta}=0, & \delta_{1}=-\delta_{2}= \pm 1, \\ k_{\delta}=1, & \delta_{1}=\delta_{2}= \pm 1 .\end{cases}
$$

Thereby, the form of the differential cross section of scattering of an electron by an electron considerably depends on the direction of rotation of vectors of the field strength of the waves. Thus, for the opposite directions of rotation of strength $\left(\delta_{1}=-\delta_{2}= \pm 1 \rightarrow k_{\delta}=0\right)$ the cross section consists of two summands corresponding to emission and absorption of photons of the same wave. If directions of rotation of vectors of the field strength of laser waves coincide $\left(\delta_{1}=\delta_{2}= \pm 1 \rightarrow k_{\delta}=1\right)$, then the cross section consists of four summands; two another summands correspond to absorption of a photon from one of the waves and emission of a photon of another wave. If electrons scatter into the angle $\theta_{1}^{\text {res }}$, then we have one summand corresponding to absorption and emission of photons of the first wave plus the second summand corresponding to absorption of a photon from the first wave but emission of a photon of the second wave. If electrons scatter into the angle $\theta_{2}^{\text {res }}$, then we have one summand corresponding to absorption and emission of photons of the second wave plus the second summand corresponding to absorption of a photon from the second laser wave but emission of a photon of the first laser wave (see, the expression for the function $f_{2}(78)$ ).

Dependence of the function $f^{\text {res }}(74)$ on parameters $\beta_{n}$ (71) determines both the magnitude and form of resonant peaks in the process cross section. It is obviously that scattering occurs into one of resonant angles $\theta \simeq \theta_{1}^{\text {res }}$ or $\theta \simeq \theta_{2}^{\text {res }}$, then the corresponding parameter $\beta_{1}$ or $\beta_{2}$ equals zero. At that the function $f^{\text {res }}$ possesses a large but finite value [17,45-49], equal to

$$
f^{\text {res }}=\omega_{1}^{2} \tau_{1}^{2}\left[\frac{1}{\left(\theta_{1}^{\text {res }}\right)^{4}} f_{1}(\rho, 0)+\frac{1}{\left(\theta_{2}^{\text {res }}\right)^{4}} f_{2}(\rho, 0)\right],
$$

unlike the case of a plane monochromatic wave, when $f^{r e s} \rightarrow \infty$ is correct in this case. Dependence of the function $f^{\text {res }}$ (74) on the scattering angle for the envelope functions (54) is shown on the Figure 3.

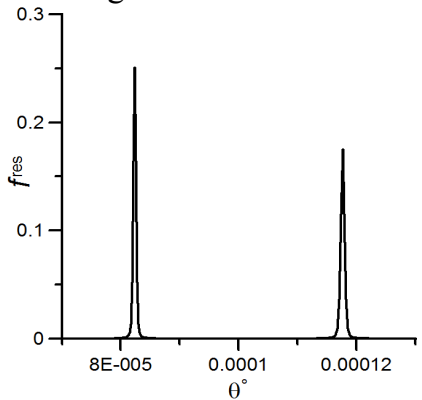

Figure 3. Dependence of the function $f^{\text {res }}$ (74) on the electron scattering angle in the units $\eta_{01}^{4}\left(\omega_{1} \tau_{1}\right)^{2}\left(\theta_{1}^{\text {res }}\right)^{-4}$ for the field parameters: $\omega_{1}=2.1 \mathrm{eV}, \omega_{2}=3 \mathrm{eV}, \eta_{01}=\eta_{02}=0.1, \delta_{1}=-\delta_{2}= \pm 1, \tau_{1}=\tau_{2}$, $\rho=5$.

Figure 3 demonstrates that there two resonant peaks in the cross section of scattering of an electron by an electron in the field of two pulsed waves. Each of peaks corresponds to 
scattering into the angles close to the resonant ones. The distance between the peaks is determined by the difference of frequency of the external laser waves. If waves' frequency differs too little, then peaks are blended and we get the single wave case [24].

Moreover, the difference of peak altitude also is determined by the ratio of frequency of the field of two pulsed laser waves.

We may integrate differential cross-section (73) within the narrow range of scattering angles near the resonance (61).

Integration over the scattering angle $\theta$ is substituted by integration over parameters $\beta_{n}$ (71). For the case of the opposite directions of rotation of the field strength ( $\delta_{1}=-\delta_{2}= \pm 1$ ) we obtain the expression for the differential cross section of resonant scattering of an electron by an electron in the field of two pulsed laser waves within the interference region in the form:

$$
\frac{d \sigma_{\left(\delta_{1}=-\delta_{2}= \pm 1\right)}^{r e s}}{d \varphi_{f}}=\frac{(2 \pi)^{4} r_{e}^{2} m^{6}}{4 \mathbf{p}^{4} E_{i}^{2}} \Phi_{-}^{r e s},
$$

where $d \varphi_{f}$ is the element of the azimuthal angle of particles escape in the inertia system. The function $\Phi_{-}^{\text {res }}$ is determined by the expression:

$$
\begin{gathered}
\Phi_{-}^{r e s}=\frac{\left(\eta_{01}^{(1)} \eta_{01}^{(2)}\right)^{2}}{\Gamma_{1}} \Upsilon_{1}+\frac{\left(\eta_{02}^{(1)} \eta_{02}^{(2)}\right)^{2}}{\Gamma_{2}} \Upsilon_{2}, \\
\Gamma_{n}=\frac{1}{\sqrt{2 \pi}} \frac{\left(\theta_{n}^{r e s}\right)^{2}}{\omega_{1} \tau_{1}}, \\
\Upsilon_{1}(\rho)=\frac{3}{\rho} \int_{0}^{\rho} d \phi\left\{e^{-(2 \phi)^{2}}+e^{-\left(2 \frac{\tau_{2}}{\tau_{1}} \phi\right)^{2}}\left(\frac{\eta_{02}^{(2)}}{\eta_{01}^{(1)}}\right)^{2}\right\} \times \\
(\operatorname{erf}(2 \sqrt{2} \phi)+1 / 3), \\
\Upsilon_{2}(\rho)=\frac{1}{\rho} \int_{0}^{\rho} d \phi\left\{e^{-\left(2 \frac{\tau_{2}}{\tau_{1}}\right)^{2}}+e^{-(2 \phi)^{2}}\left(\frac{\eta_{01}^{(2)}}{\eta_{02}^{(1)}}\right)^{2}\right\} \times \\
\left(\frac{\tau_{2}}{\tau_{1}}\left(\frac{\tau_{2}}{\tau_{1}}+2\right) \operatorname{erf}\left(2 \sqrt{2} \frac{\tau_{2}}{\tau_{1}} \phi\right)+1\right) .
\end{gathered}
$$

The quantities $\Gamma_{n}$ (85) are the resonance transit widths [49]. It is easy to notice that

$$
\Gamma_{n} \sim\left(\frac{\omega_{n}}{|\mathbf{p}|}\right)^{2} \frac{1}{\omega_{1} \tau_{1}} \sim 10^{-6},
$$

and to estimate the ratio of the widths $\Gamma_{1}$ and $\Gamma_{2}$ :

$$
\frac{\Gamma_{1}}{\Gamma_{2}} \sim\left(\frac{\omega_{1}}{\omega_{2}}\right)^{2} .
$$

Broadening of the resonant peak in particle scattering in the pulsed-wave field is specified by both the wave finite duration and observation time. It was noticed in works by Ritus and Nikishov [2] that observation of a process during $\mathrm{N}$ wave periods results in fulfillment of the energy conservation law with an accuracy $N^{-1}$ [14]. Consequently, broadening of resonance peaks at the expense of wave finite duration in scattering of an electron by an electron in the field of two pulsed laser waves appears for an arbitrary values of the parameter $\rho$.

For the case of coincident directions of rotation of the field strength $\left(\delta_{1}=+\delta_{2}= \pm 1\right)$ we obtain the expression for the differential cross section of resonant scattering of an electron by an electron in the field of two pulsed laser waves within the interference region in the form:

$$
\begin{gathered}
\frac{d \sigma_{\left(\delta_{1}=\delta_{2}=11\right)}^{r e s}}{d \varphi_{f}}=\frac{(2 \pi)^{4} r_{e}^{2} m^{6}}{4 \mathbf{p}^{4} E_{i}^{2}} \Phi_{+}^{r e s}, \\
\Phi_{+}^{\text {res }}=\frac{\left(\eta_{01}^{(1)} \eta_{01}^{(2)}\right)^{2}}{\Gamma_{1}} \Xi_{1}(\rho)+\frac{\left(\eta_{02}^{(1)} \eta_{02}^{(2)}\right)^{2}}{\Gamma_{2}} \Xi_{2}(\rho), \\
\Xi_{1}(\rho)= \\
\frac{3}{\rho} \int_{0}^{\rho} d \phi \cdot e^{-(2 \phi)^{2}}(\operatorname{erf}(2 \sqrt{2} \phi)+1 / 3), \\
\Xi_{2}(\rho)=\frac{1}{\rho} \int_{0}^{\rho} d \phi \cdot e^{-\left(2 \frac{\tau_{2}}{\tau_{1}} \phi\right)^{2}} \times \\
\times\left(\frac{\tau_{2}}{\tau_{1}}\left(\frac{\tau_{2}}{\tau_{1}}+2\right) \operatorname{erf}\left(2 \sqrt{2} \frac{\tau_{2}}{\tau_{1}} \phi\right)+1\right) .
\end{gathered}
$$

Transit widths $\Gamma_{n}$ in (91) are determined by (85).

The significant issue is the influence of finite duration of laser wave on resonant behavior of scattered electrons. The assertion that the pulsewidth must exceed the time required for realization of the Compton scattering of an external field photons by each of the electrons is to be taken into consideration. If this condition is not satisfied than particles do not have time to interact with the wave under the resonant conditions [6-7]. Consequently the following correlation is valid for the pulse duration

$$
\tau_{n} \gtrsim \frac{1}{\Gamma_{n}^{r a d}}
$$

where

$$
\Gamma_{n}^{r a d} \sim \alpha\left(\eta_{0 n}^{(j)}\right)^{2} \frac{\omega_{n} \kappa_{j}}{E_{j}}
$$

is the radiation width, which is determined by the total probability (per time unit) of the Compton scattering of an external field photon on each of electrons with the relative momentum $\mathbf{p}$.

Inconsequence of the expression (94) we derive that the value of the parameter $\omega_{n} \tau_{n}$ must satisfy the condition

$$
\omega_{n} \tau_{n} \gtrsim \frac{1}{\alpha\left(\eta_{0 n}^{(j)}\right)^{2}} \frac{E_{j}}{\kappa_{j}} .
$$


Due to correlation (96) one can define that for the frequency optical range and the external pulsed wave intensity range (10) the pulsewidth meets the condition (the cases of particle ultrarelativistic energy when their movement within the narrow cone with an external field photon are excluded from consideration)

$$
\tau_{n} \gtrsim 10^{-12} s .
$$

Using expressions (73)-(78) one can estimate the ratio of the resonant cross section of scattering of an electron by an electron in the field of two pulsed laser waves (within the interference region) to the cross section in the absence of the external field. Thereby, for the nonrelativistic case, the waves' optical frequency and the number of oscillation of the field amplitude at the characteristic pulse duration $\omega_{1} \tau_{1} \sim 10^{2}$ this ratio is one order of the magnitude.

Thus, experimental study of resonant scattering of an electron by an electron may be verified in the fields produced by picosecond pulsed lasers which generate radiation within the optical frequency range. Parameters of the pulsed laser can be, for example, the following: the photon energy $\hbar \omega_{n}=2.35 \mathrm{eV}$, the pulse duration $\tau_{n}=1.5 \mathrm{ps}$, the strength of the electric field $F_{0 n}=6 \cdot 10^{9} \mathrm{~V} / \mathrm{cm}$, the parameter $\rho=\sqrt{2}$ for the electron energy $E_{j}=2.5 \mathrm{keV}$. Such scientific facilities are employed in the SLAC (Princeton Rochester, SLAC, Tennessee collaboration) and Brookhaven National Laboratory (USA) research centers, and also in the frame of the FAIR Project (Darmstadt, Germany).

\section{Conclusion}

Study of resonant scattering of an electron by an electron in the field of two pulsed laser waves within the interference region results in following conclusion:

1. Resonant scattering of an electron by an electron in the field of two pulsed laser waves within the interference region appears under process certain geometry. Thus, for electron nonrelativistic energy it corresponds to the case when the particle ingoing angle is close to $\pi / 2$ in the frame of the reference related to the center of inertia of initial particles.

2. The form of resonant cross section of the studied process considerably depends on the direction of rotation of vectors of the field strength of the waves. Thus, for opposite directions of rotation of the strength $\left(\delta_{1}=-\delta_{2}= \pm 1\right)$ the cross section consists of two summands corresponding to emission and absorption of photons of the same wave. If directions of rotation of vectors of the field strength of laser waves coincide ( $\delta_{1}=\delta_{2}= \pm 1$ ), then processes with absorption of a photon from one of the waves and emission of a photon of another wave are allowed.

3.The resonant differential cross section of scattering of nonrelativistic electrons by each other in the field of two pulsed laser waves of the optical frequency (10) within the interference region is approximately ten times greater in the magnitude than the corresponding cross section in the external field absence.

\section{REFERENCES}

[1] G.A. Mourou, T. Tajima, S.V. Bulanov. Optics in the relativistic regime, Review of Modern Physics, Vol. 78, 309, 2006.

[2] A.I. Nikishov, V.I. Ritus. Quantum electrodynamics of phenomena in an intense field, Trudy FIAN, Vol. 111, 1-279, 1979 (in Russian).

[3] N.B. Delone. Interaction of Laser Radiation with Matter, Nauka, Moscow, 1989 (in Russian).

[4] M.V. Fedorov. An Electron in a Strong Light Field, Nauka, Moscow, 1991 (in Russian).

[5] S.P. Roshchupkin, A.I. Voroshilo, Resonant and Coherent Effects of Quantum Electrodynamics in the Light Field, Naukova Dumka, Kiev, 2008 (in Russian).

[6] S.P. Roshchupkin, A.A. Lebed'. Effects of Quantum Electrodynamics in the Strong Pulsed Laser Fields, Naukova Dumka, Kiev, 2013 (in Russian).

[7] S.P. Roshchupkin, A.A. Lebed', E. A. Padusenko, A. I. Voroshilo. Quantum electrodynamics resonances in a pulsed laser field, Laser Physics, Vol. 22, № 6, 1113-1144, 2012.

[8] S.P. Roshchupkin, A.A. Lebed', E.A. Padusenko. Nonresonant quantum electrodynamics processes in a pulsed laser field, Laser Physics, Vol. 22, № 10, 1-34, 2012.

[9] F. Ehlotzky, K. Krajewska, J.Z. Kaminski. Fundamental processes of quantum electrodynamics in laser fields of relativistic power, Reports of Progressive Physics, Vol. 72, № 4, 046401, 2009.

[10] C. Bula, K. T. McDonald, E. J. Prebys, C. Bamber, S. Boege, T. Kotseroglou, A. C. Melissinos, D. D. Meyerhofer, W. Ragg, D. L. Burke, R. C. Field, G. Horton-Smith, A. C. Odian, J. E. Spencer, D. Walz, S. C. Berridge, W. M. Bugg, K. Shmakov, and A. W. Weidemann, Observation of nonlinear effects in Compton scattering, Physical Review Letters, Vol. 76, № 17, 3116-3119, 1996.

[11] D. L. Burke, R. C. Field, G. Horton-Smith, J. E. Spencer, D. Walz, S. C. Berridge, W. M. Bugg, K. Shmakov, A. W. Weidemann, C. Bula, K. T. McDonald, E. J. Prebys, C. Bamber, S. J. Boege, T. Koffas, T. Kotseroglou, A. C. Melissinos, D. D. Meyerhofer, D. A. Reis, and W. Ragg, Positron production in multiphoton light by light scattering, Physical Review Letters, Vol. 79, № 9, 1626-1629, 1997.

[12] V Bagnoud, B Aurand, A Blazevic, S Borneis, C Bruske, B Ecker, U Eisenbarth, J Fils, A Frank, E Gaul, P Neumayer, E Onkels, D Reemts, O Rosmej, M Roth, T Stoehlker, A Tauschwitz, B Zielbauer, D Zimmer, K Witte, Commissioning and early experiments of the PHELIX facility, Applied Physics B, Vol. 100, № 1, 137-150, 2010.

[13] B. Zielbauer, T. Kuehl, B. Aurand, V. Bagnoud, B. Ecker, U. Eisenbarth, D. C. Hochhaus, P. Neumayer, D. Zimmer, K. 
Cassou, S. Daboussi, O. Guilbaud, J. Habib, S. Kazamias, D. Ros, J. Seres, C. Spielmann, X-ray laser developments at PHELIX, Springer Proceedings in Physics, Vol. 136, 31-38, 2011.

[14] N.B. Narozhny, M.S. Fofanov. Photon emission by an electron in a collision with a short focused laser pulse, Journal of Experimental and Theoretical Physics, Vol. 83, 14-24, 1996.

[15] K. Krajewska, J.Z. Kaminski. Compton process in intense short laser pulses, Physical Review A, Vol. 85, 062102, 2012.

[16] K. Krajewska, J.Z. Kaminski. Phase effects in laser-induced electron-positron pair creation, Physical Review A, Vol. 85, 043404, 2012.

[17] S.P. Roshchupkin, A.A. Lebed', E.A. Padusenko, A.I Voroshilo, Resonant Effects of Quantum Electrodynamics in the Pulsed Light Field, Quantum Optics and Laser Experiments, edited by S. Lyagushyn, Intech, Rijeka, 2012, 107-156.

[18] D. Volkov. Übereine Klasse von Lösungen der Diracschen Gleichung, Zeit. Physics, Vol. 94, 250, 1935.

[19] V.B. Berestetskii, E.M. Lifshitz, L.P. Pitaevskii. Quantum Electrodynamics, Nauka, Moscow, 1980 (in Russian).

[20] C. Möller. Zur theorie des durchgangs schneller elektronen durch materie, Ann. Physics, Vol. 406, № 5, 531-585, 1932.

[21] R.V. Karapetian, M.V. Fedorov. Influence of an intense electromagnetic wave on stimulated bremsstrahlung of electrons, Quantum Electronics, Vol. 4, № 10, 2203-2215. 1977 (in Russian).

[22] O.I. Denisenko, S.P. Roshchupkin. Non-resonance electron scattering in the field of two plane light waves, Physica Scripta, Vol. 50, 339-342, 1994.

[23] R.L. Gorodnitskii, S.P. Roshchupkin. Induced bremsstrahlung process for a relativistic electron colliding with a nucleus in a field of two electromagnetic waves of arbitrary intensities and frequencies, Laser Physics, Vol. 2, № 4, 602-608, 1992.

[24] S.P. Roshchupkin. The effect of a strong light field on the scattering of an ultrarelativistic electron by a nucleus, Journal of Theoretical and Experimental Physics, Vol. 82, № 2, $177-180,1996$

[25] S.P. Roshchupkin. The interference effect in the scattering of an electron by a nucleus in the field of two plane electromagnetic waves, Journal of Theoretical and Experimental Physics, Vol. 79, № 1, 54-63, 1994.

[26] I.M. Ternov, V.G. Bagrov, Yu.I. Klimenko. Motion and radiation of an electron exhibiting a vacuum magnetic moment in the field of a plane electromagnetic wave, Russian Physics Journal, Vol. 11, № 2, 29-33, 1968.

[27] Yu.I. Klimenko, A.I. Hudomyasov. Induced radiation of fermi particles with anomalous magnetic moment in the field of two electromagnetic waves, Soviet Physics Journal, Vol. 15, № 11, 1593-1597, 1972 .

[28] Yu.I. Klimenko, V.V. Kulish, A.I. Hudomyasov. Bremsstrahlung of a neutral fermi particle in the field of a planeelectromagneticwave, Soviet Physics Journal, Vol. 18, № 10, 1410-1414, 1975 .
[29] Yu.I. Klimenko, V.V. Kulish, I.I. Fedosov, A.I. Hudomyasov. A charge in the field of two plane electromagnetic waves having arbitrary polarizations, Soviet Physics Journal, Vol. 19, № 4, 521-522, 1976.

[30] S.P. Roshchupkin, O.B. Lysenko. Nonresonant spontaneous bremsstrahlung in scattering of a relativistic electron by a nucleus in the field of two light waves, Laser Physics, Vol. 9, № 2, 494-503, 1999 .

[31] S.P. Roshchupkin, O.B. Lysenko. Spontaneous interference bremsstrahlung effect in the scattering of a relativistic electron by a nucleus in the field of two light waves, Journal of Theoretical and Experimental Physics, Vol. 89, № 4, 647-663, 1999.

[32] S.P. Roshchupkin, A.I. Voroshilo. Interference effect in the emission of a spontaneous photon by an electron in the field of two light waves, Laser Physics, Vol. 7, № 2, 466-472, 1997.

[33] A.V. Borisov, O.G. Goryaga, V.Ch. Zhukovskii, A.A. Sokolov. Production of electron-positron pairs on nuclei in the field of a bichromatic plane electromagnetic wave, Soviet Physics Journal, Vol. 21, № 9, 1136-1142, 1978.

[34] S. P. Roshchupkin. Interference effect in photoproduction of electron-positron pairs on a nucleus in the field of two light waves, Physics of Atomic Nuclei, Vol. 64, № 2, 243-252, 2001 .

[35] S. P. Roshchupkin. Resonant spontaneous bremsstrahlung of an electron in the field of the nucleus and two light waves, Laser Physics, Vol. 12, № 2, 498-503, 2002.

[36] S.P. Roshchupkin, V.A. Tsybul'nik. The light amplification effect in the Coulomb scattering of nonrelativistic electrons in a two-mode laser field, Laser Physics Letters, Vol. 3, № 7, $362-368,2006$

[37] S.P. Roshchupkin, A.I. Voroshilo. Stimulated bremsstrahlung in electron-nucleus scattering in a multifrequency electromagnetic field, Laser Physics, Vol. 7, № 3, 873-884, 1997.

[38] A.I. Voroshilo and S.P. Roshchupkin, Formation of an electron-positron pair by a quantum in a multifrequency electromagnetic field, Laser Physics, Vol. 10, № 5, 1078-1085, 2000

[39] V.P. Oleinik. Resonant effects in the field of intense laser beam, Journal of Experimental and Theoretical Physics, Vol. 52, 1049-1067, 1967. [Soviet Physics Journal of Experimental and Theoretical Physics, Vol. 25, 697, 1967].

[40] V. P. Oleinik. Resonant effects in the field of intense laser beam II, Journal of Experimental and Theoretical Physics, Vol.53, 1997, 1967. [Soviet Physics Journal of Experimental and Theoretical Physics, Vol. 26, 1132 (1967)].

[41] S.P. Roshchupkin. Resonance scattering of an electron by an electron in the field of a light wave: general relativistic case, Laser Physics, Vol. .4, № 1, P.139-147, 1994.

[42] S.P. Roshchupkin. Resonant effect in collisions of relativistic electrons in the field of light wave, Laser Physics, Vol. 6, № 5, $837-858,1996$

[43] J. Bös, W. Brock, H. Mitter, T. Schoott. Resonances and intensity-dependent shifts of the Möller cross section in a strong laser field, Journal of Physics A, Vol. 12, № 5, 715-731, 
1979.

[44] P. Panek, J.Z. Kaminski, F. Ehlotzky. Analysis of resonances in Möller scattering in a laser field of relativistic radiation power, Physical Review A, Vol. 69, 012404, 2004.

[45] E.A. Padusenko,S.P. Roshchupkin. Resonant scattering of a lepton by a lepton in the pulsed light field, Laser Physics, Vol. 20, № 12, 2080-2091, 2010.

[46] A.I. Voroshilo, S.P. Roshchupkin. Resonant scattering of a photon by an electron in the field of a circularly polarized electromagnetic wave, Laser Physics Letters, Vol. 2, №. 4, 184-189, 2005.

[47] A.A. Lebed', S.P. Roshchupkin. Resonant electron-positron pair photoproduction on a nucleus in a pulsed light field, Journal of Experimental and Theoretical Physics, Vol. 113, № 1, 46-54, 2011.

[48] A.I. Voroshilo, S.P. Roshchupkin, V.N. Nedoreshta. Resonant scattering of a photon by an electron in the presence of the pulsed laser field, Laser Physics, Vol. 21, № 9, 1675-1687, 2011.

[49] A.A. Lebed', S.P. Roshchupkin. Resonant spontaneous bremsstrahlung by an electron scattered by a nucleus in the field of a pulsed light wave, Physical Review A, Vol. 81, 033413, 2010.

[50] A.A. Lebed', S.P. Roshchupkin. The influence of a pulsed light field on the electron scattering by a nucleus, Laser Physics Letters, Vol. 5, № 6, 437-445, 2008.
[51] A.I. Voroshilo, E.A. Padusenko, S.P. Roshchupkin. One-photon annihilation of an electron-positron pair in the field of pulsed circularly polarized light wave, Laser Physics, Vol. 20, № 7, 1679-1685, 2010.

[52] A.A. Lebed', S.P. Roshchupkin. Nonresonant spontaneous bremsstrahlung by a relativistic electron scattered by a nucleus in the field of pulsed light wave, The European Physics Journal D, Vol. 53, № 1, 113-122, 2009.

[53] A.A. Lebed', S.P. Roshchupkin. Spontaneous bremsstrahlung effect in the nonrelativistic electron scattering by a nucleus in the field of pulsed light wave, Laser Physics Letters, Vol. 6, № 6, 472-479, 2009.

[54] E.A. Padusenko, S.P. Roshchupkin, A.I. Voroshilo. Nonresonant scattering of relativistic electron by relativistic muon in the pulsed light field, Laser Physics Letters, Vol. 6, № 3, 242-251, 2009.

[55] E.A. Padusenko, S.P. Roshchupkin, A.I. Voroshilo. Nonresonant scattering of nonrelativistic electron by nonrelativistic muon in the pulsed light field, Laser Physics Letters, Vol. 6, № 8, 616-623, 2009.

[56] F.V. Bunkin and M.V. Fedorov, Bremsstrahlung in a Strong Radiation Field, Soviet Physics Journal of Experimental and Theoretical Physics, Vol. 22, 844-847, 1966.

[57] S.P. Roshchupkin, V.A. Tsubyl'nik, A.N. Chmirev. Probability of multiphoton processes in phenomena of a quantum electrodynamics in a strong light field, Laser Physics, Vol. 10, № 6, 1231-1248, 2000. 